\title{
Afrontamiento de problemas de salud en personas muy mayores
}

\author{
Ana B. Navarro ${ }^{1 *}$ y Belén Bueno ${ }^{2}$ \\ 1 Escuela Universitaria de Magisterio de Zamora (España) \\ 2 Facultad de Psicologia, Salamanca (España)
}

\begin{abstract}
Resumen: En este estudio se evalúan las estrategias de afrontamiento ante problemas de salud en la vejez avanzada y su contribución sobre varios resultados de funcionamiento. 159 personas mayores de 75 años no institucionalizadas identificaron su problema de salud reciente más importante, las estrategias empleadas para hacerle frente, su percepción de autoeficacia en el manejo del problema y su grado de satisfacción vital. Los resultados confirman la utilización de variadas estrategias, siendo la activacomportamental resolver el problema la más empleada. Además, junto a las estrategias de afrontamiento activas, tanto cognitivas como comportamentales, los análisis correlacionales indican que las personas muy mayores recurren a modos de afrontamiento pasivos y evitativos. Por otra parte, los análisis de regresión múltiple ponen de manifiesto que utilizar acciones directas y racionales para solucionar los problemas de salud predice la autoeficacia en el manejo del problema y protege la satisfacción vital en esta etapa. Estos resultados confirman que las personas muy mayores conservan la capacidad para manejar eficazmente sus problemas de salud y, al mismo tiempo, mantener su bienestar, aportando evidencia sobre el papel adaptativo del afrontamiento en la vejez avanzada.

Palabras clave: problemas de salud; afrontamiento; personas muy mayo-
\end{abstract} res; resiliencia; estudio ex post facto.

\section{Introducción}

El envejecimiento de los muy mayores está suscitando gran interés dentro de la comunidad científica debido a dos razones fundamentales: las personas muy mayores $(75+)$ constituyen el sector de población que más ha aumentado en los últimos años y que más va a crecer en las próximas décadas (UNFPA, 2012), pero se conoce muy poco específicamente sobre este grupo de edad. En tanto que "nueva etapa" del ciclo vital, la vejez avanzada plantea numerosos interrogantes a los que algunos investigadores han empezado a responder (p. ej., Baltes y Mayer, 1999; Martin, Rott, Poon, Courtenay y Lehr, 2001).

La salud y el valerse por uno mismo son dos de los aspectos más importantes de la vida para las personas mayores (Thomae, 2002), siendo las amenazas a la salud y a la autonomía personal sus preocupaciones centrales (Montorio, Nuevo, Márquez, Izal y Losada, 2003). Sin embargo, a pesar de las enfermedades crónicas y discapacidades, se ha constatado que, al menos hasta fases terminales de la vida, no existe un declive general relacionado con la edad en indicadores de calidad de vida subjetiva, tales como satisfacción vital, autoestima, o control personal, ni evidencia epidemiológica consistente de una incidencia creciente en depresión (p. ej., Diener, Ng, Harter y Arora, 2010; Sonnenberg et al., 2013). Estos hallazgos han impulsado la investigación sobre resiliencia psicológica en la vejez, entendida como la capacidad para mantener o recuperar la salud y el bienestar frente a la

* Dirección para correspondencia [Correspondence address]:

Belén Bueno Martínez. Facultad de Psicología. Avda. de la Merced, 109

131.37005 Salamanca (España).E-mail: bbueno@usal.es
Title: Coping with health problems in very old people.

Abstract: This paper assesses the strategies for coping with health problems in advanced old age and their contribution in terms of several performance results. 159 people aged 75 or over and living at home identified their most recent health problem, the strategies used to deal with it, their perception of self-efficacy in handling the problem and their degree of satisfaction with life. The results confirm the use of a range of strategies, with the active-behavioural approach to solving the problem being the one most widely used. In addition, together with active coping strategies of both a cognitive and behavioural nature, correlational analyses indicate that very old people resort to passive and avoidance coping methods. Furthermore, multiple regression analyses highlight the fact that the use of direct and rational actions for solving health problems predicts self-efficacy in dealing with the problem and protects satisfaction with life at this stage. These results confirm that very old people retain the ability to deal effectively with their health problems and, at the same time, uphold their well-being, providing evidence of the adaptive role of coping in very old age.

Key words: health problems; coping; advanced old age; resilience; ex post facto study.

acumulación de situaciones adversas (Ong, Bergeman y Boker, 2010; Staudinger, Freund, Linden y Maas, 1999).

Se piensa que la resiliencia psicológica es un proceso dinámico que resulta de la acción de múltiples factores protectores que impiden que se produzcan cambios en los niveles de satisfacción (para una revisión reciente sobre la resiliencia, véase Wu et al., 2013). Entre otros, el afrontamiento se postula como un factor protector por ser un recurso interno que las personas mayores ponen en marcha para hacer frente a las situaciones de estrés (Aldwin, 2007; Aldwin y Park, 2004; Meléndez, Mayordomo, Sancho y Tomás, 2012; Taylor y Stanton, 2007; Yancura y Aldwin, 2008). Específicamente, el afrontamiento de los problemas de salud se entiende como el modo en que la persona maneja las pérdidas en las funciones corporales o las discapacidades derivadas de enfermedades físicas crónicas.

En el ámbito de la salud, gran parte de la investigación sobre afrontamiento en la vejez se ha centrado en patologías concretas, con los procesos de adaptación al cáncer ocupando la mayor parte de la bibliografía sobre el afrontamiento de las enfermedades crónicas. Varias investigaciones han planteado los efectos positivos de estrategias como la reevaluación positiva en la adaptación al cáncer, en detrimento de las estrategias de evitación (Carver, 2004; Chida y Hamer, 2008; Dunkel-Schetter, Feinstein, Taylor y Falke, 2000; Holland y Holahan, 2003; Urcuyo, Boyers, Carver y Antoni, 2005; Zaza, Sellick y Hillier, 2005). También, la aceptación de la enfermedad, respuesta de afrontamiento más utilizada por mujeres con cáncer de mama durante el año posterior a la intervención quirúrgica, se asocia con bajos niveles de afecto negativo y con un mayor ajuste (McKenna, Zevon, Corn y Rounds, 1999). Asimismo, ante otro tipo de enfer- 
medades como la artritis reumatoide, la búsqueda de información predice un incremento del afecto positivo (Franks y Roesch, 2006; Revenson, Abraído-Lanza, Majerovitz y Jordan, 2005). Estudios sobre enfermos con problemas cardiovasculares han confirmado que las estrategias evitativas se asociaban con una menor calidad de vida y las estrategias activas se relacionaban con altos niveles de autoestima, satisfacción con la vida y seguimiento de las prescripciones médicas (Prokopcakova, 2004).

Otra línea de investigación relevante se ha centrado en la identificación de las circunstancias vitales estresantes más frecuentes en dominios vitales diferentes y en el análisis de los patrones de afrontamiento de esos tipos de sucesos. Los resultados más importantes de estos estudios indican que las estrategias de afrontamiento más utilizadas frente a los problemas de salud son las de aproximación cognitiva y comportamental (p. ej., Aldwin, 2007), si bien cuando se comparan con otros sucesos estresantes, los problemas de salud es más probable que eliciten estrategias de afrontamiento centradas en las emociones o estrategias de evitación (p. ej., Moos, Brennan, Schutte y Moos, 2006). No obstante, hasta la actualidad apenas existen trabajos que hayan explorado estas cuestiones en personas muy mayores. En relación con los estudios más relevantes en este sentido, Aldwin, Sutton, Chiara y Spiro (1996) investigaron las diferencias de edad en el afrontamiento de problemas considerando un grupo de personas mayores de 75 años, pero no evaluaron los diferentes tipos de problemas por separado. Martin et al. (2001) únicamente consideraron las conductas de afrontamiento específicas ("moleculares") frente a problemas de salud de los grupos de mayores octogenarios y centenarios que investigan. Y el interesante trabajo realizado por Moos et al. (2006) evaluando las variaciones intraindividuales en el afrontamiento de sucesos de salud, interpersonales y económicos/laborales se detiene en personas de 65 años de edad.

Por otra parte, se ha demostrado que no existen procesos de afrontamiento buenos o malos para todas las situaciones. La adecuación y efectividad del afrontamiento debe considerarse dentro de su contexto. Las mismas estrategias de afrontamiento pueden contribuir a diferentes resultados y no todas son igualmente eficaces en el manejo del estrés (Meléndez et al., 2012; Thompson et al., 2010). En este sentido, son muy escasos los estudios que han incluido la evaluación de la autoeficacia en el manejo del suceso como consecuencia del afrontamiento utilizado. La limitada evidencia disponible indica que quienes se apoyan más en el afrontamiento de aproximación y menos en el evitativo es más probable que crean que manejaron bien el suceso, tanto en el caso de personas jóvenes (Harnish, Aseltine y Gore, 2000) como en personas mayores ante todo tipo de sucesos vitales negativos (Kraaij, Garnefski y Maes, 2002), pero es necesario conocer si la misma situación se mantiene en la vejez avanzada frente a diferentes problemas de salud.

Además, la mayoría de los estudios previos se ha centrado en resultados de funcionamiento negativos, tales como la depresión (Kraaij, Pruymboom y Garnefski, 2002; Rothermund y Brandtstädter, 2003), habiéndose obtenido que los adultos que utilizan más el afrontamiento evitativo y menos la reestructuración cognitiva y la resolución del problema es más probable que experimenten depresión. Si bien estos trabajos son interesantes, hasta la actualidad muy pocos estudios han considerado la vinculación en la vejez entre estrategias de afrontamiento y la satisfacción vital en tanto que resultado del funcionamiento. Únicamente Staudinger et al. (1999) y Hamarat, Thompson, Zabrucky, Steele y Matheny (2001) investigaron en esta línea asumiendo que una parte importante del estado de salud viene recogido por el nivel de satisfacción vital de la persona. En sus estudios han encontrado que el afrontamiento explica una parte importante de la satisfacción vital, que la pasividad en el manejo de los problemas no mantiene ni intensifica la satisfacción vital en la vejez avanzada, y que las personas mayores de 70 años que informaban de altos niveles de satisfacción empleaban gran número de estrategias de afrontamiento diferentes.

Tras haber identificado los estresores propios de la vejez avanzada en población no institucionalizada, con el presente estudio ex post facto (Montero y León, 2005) se pretenden averiguar los recursos cognitivos y comportamentales que utilizan las personas muy mayores para hacer frente a sus problemas en el dominio de la salud con el fin de conocer sus repertorios de afrontamiento, identificar los cambios que se producen en ellos con la edad avanzada, así como valorar el impacto que la movilización de dichos recursos personales tiene sobre determinados resultados en dicha etapa vital. Por este motivo, en este estudio se plantearon los siguientes objetivos de investigación: (a) examinar las estrategias de afrontamiento de los problemas de salud más empleadas por las personas muy mayores y las diferencias en su utilización vinculadas a la edad avanzada; (b) determinar la contribución de las estrategias de afrontamiento de los problemas de salud sobre la autoeficacia en el manejo de dichos problemas y sobre la satisfacción vital en la vejez avanzada. Las hipótesis fueron las siguientes: a) en la vejez avanzada se empleará un gran número de estrategias diferentes frente a los problemas de salud; b) a mayor edad se utilizarán menos estrategias activas y más estrategias pasivas y evitativas; c) el afrontamiento activo de los problemas de salud en la vejez avanzada contribuirá positivamente sobre la autoeficacia en su manejo, mientras que el afrontamiento pasivo y evitativo contribuirán negativamente; d) el modo en que se afrontan los problemas de salud tendrá una contribución significativa sobre la satisfacción vital en la vejez avanzada.

\section{Método}

\section{Participantes}

La muestra que participó en este estudio estuvo compuesta por 159 personas que refirieron problemas de salud en una investigación más amplia sobre calidad de vida en la vejez avanzada en la que, entre otras cosas, se preguntó so- 
bre los problemas más importantes experimentados. En dicha investigación se recogieron datos de 400 personas mayores de 75 años que vivían en la comunidad seleccionadas aleatoriamente, y se obtuvo que el $92.7 \%$ refería problemas o dificultades en su vida reciente. De todos los problemas descritos, el $42.9 \%$ correspondió a problemas de salud de la persona encuestada.

De las 159 personas que identificaron problemas de salud, 85 eran hombres y 74 mujeres. Sus edades oscilaban entre los 75 y los 104 años, con una edad media de 85.87 años $(D T=6.85)$. Todos residían en su propio domicilio. En la Tabla 1 se presentan los datos sociodemográficos de los participantes.

Tabla 1. Datos sociodemográficos de los participantes en el estudio. (En la variable nivel educativo hubo 22 casos perdidos $-13.8 \%$ - y en la variable nivel económico hubo 13 casos perdidos $-8.2 \%$-).

\begin{tabular}{lll}
\hline EDAD & $75-84$ años & 85 y más años \\
\hline SEXO & 42 & 43 \\
Hombre & 33 & 41 \\
Mujer & & \\
\hline NIVEL EDUCATIVO & $46(48.4 \%)$ & $20(47.6 \%)$ \\
Bajo & $49(51.6 \%)$ & $22(52.4 \%)$ \\
Medio-Alto & $34(42.5 \%)$ & $46(57.5 \%)$ \\
\hline NIVEL ECONÓMICO & $36(54.4 \%)$ & $30(45.5 \%)$ \\
Bajo &
\end{tabular}

Nota: Las categorías de edad y sexo se utilizaron como criterio de selección de la muestra. Las variables nivel educativo y nivel económico se establecieron ad-hoc y se unificó una categoría medio-alto al existir muy pocos participantes de nivel alto.

\section{Instrumentos}

Para evaluar el afrontamiento, se aplicó la Escala de Estilos y Estrategias de Afrontamiento -E3A- de Fernández-Abascal (1997), mediante la que se pedía identificar en qué medida cada una de las frases describía formas más o menos habituales de enfrentarse a su problema. Consta de 72 ítems que evalúan 18 estrategias de afrontamiento diferentes (cada una de ellas se compone de 4 ítems). Dichas estrategias se agrupan en tres dimensiones básicas: el método empleado en el afrontamiento, mediante el que se diferencian estrategias activas, pasivas y de evitación; la focalización del afrontamiento, según la cual se distinguen estrategias orientadas a modificar la evaluación inicial de la situación, dirigidas al problema y dirigidas a la emoción; y la actividad movilizada en el afrontamiento, que permite diferenciar estrategias cognitivas y conductuales.

Las estrategias de afrontamiento identificadas mediante la escala E3A son las siguientes: 1- Reevaluación positiva, que se refiere a las estrategias de afrontamiento activo orientadas a crear un nuevo significado de la situación problema, intentando extraer todo lo positivo que tenga la situación; 2- Reacción depresiva, por la que la persona se siente desbordada por la situación, es pesimista sobre los resultados que espera de ella y se siente incapaz de afrontar el problema; 3- Negación, que significa la no aceptación del problema y su evitación por distorsión o desfiguración del mismo al valorarlo; 4- Planificación, estrategia claramente centrada en el problema que se refiere a la movilización de estrategias de afrontamiento para alterar la situación realizando una aproximación analítica y racional al problema; 5- Conformismo, que implica una aceptación de la situación tal y como es causada por la percepción de falta de control respecto a la situación-problema; 6- Desconexión cognitiva, definida como la tendencia a la utilización de pensamientos distractores que ayuden a olvidarse del problema; 7- Desarrollo personal, que incluye elementos sobre la consideración del problema de una manera relativa, de autoestímulo y de un positivo aprendizaje de la situación, centrándose sobre todo en el desarrollo personal; 8- Control emocional, referido a la movilización de recursos orientados a regular y ocultar los propios sentimientos; 9- Distanciamiento, que implica la supresión cognitiva de los efectos emocionales que el problema genera; 10- Supresión de actividades distractoras, que se refiere a eliminar todas aquellas actividades o tareas que puedan estar interfiriendo en el afrontamiento de la situación-problema, para centrarse activamente en la búsqueda de información para valorarla; 11- Refrenar el afrontamiento consiste en aplazar cualquier tipo de comportamiento para solucionar la situación hasta que se disponga de mayor información respecto a ella; 12- Evitar el afrontamiento implica no hacer nada en previsión de que cualquier tipo de actuación puede empeorar la situación o por valorar el problema como irresoluble; 13- Resolver el problema es el intento activo de manejar o solucionar el problema y supone decidir una acción directa y racional para solucionar las situaciones problema; 14- Apoyo social al problema se refiere a la búsqueda de consejo, asistencia e información en los otros sobre cómo resolver el problema; 15- Desconexión comportamental consiste en la evitación de cualquier tipo de comportamiento para resolver el problema; 16- Expresión emocional, en la que se canaliza el afrontamiento hacia las manifestaciones dirigidas a otras personas de las emociones causadas por el problema; 17- Apoyo social emocional, por la cual se busca en los demás apoyo y comprensión para la situación emocional en la que uno se halla inmerso; 18- Respuesta paliativa, caracterizada por incluir en su afrontamiento elementos que buscan la evitación de la tensión ocasionada por la situación estresante, realizando determinados comportamientos, tales como buscar sentirse mejor con la religión, viendo la televisión o comiendo.

Los sujetos responden a cada ítem utilizando una escala tipo Likert graduada de 0 a 3 , donde 0 indica que quien responde nunca utiliza esa estrategia y 3 indica que la emplea siempre. La consistencia interna para la escala total fue de .76, resultado similar al obtenido por Tobin, Holroyd, Reynolds y Wigal (1989) con su "Coping Strategies Inventory".

Para evaluar la autoeficacia en el manejo de los problemas de salud, se pidió a los participantes que indicaran cómo de efectivo creían que había sido su manejo del problema. Las personas respondían en una escala tipo Likert graduada de 0 a 3 (desde $0=$ nada efectivo hasta $3=$ muy efectivo). 
El grado de bienestar se evaluó con la Escala de Satisfacción con la Vida -SWLS- desarrollada por Diener, Emmons, Larsen y Griffin (1985). Está formada por 5 ítems relacionados con aspectos importantes de la vida sobre los que hay que indicar el grado de acuerdo o desacuerdo. Los 5 ítems forman un único factor. Los participantes respondían utilizando una escala tipo Likert graduada de 1 a 4 (desde $1=$ total desacuerdo hasta $4=$ total acuerdo). El rango de puntuaciones va desde una mínima satisfacción con la vida (5) a muy alta (20). La consistencia interna de la escala SWLS fue de .71.

\section{Procedimiento}

Para realizar la investigación amplia sobre calidad de vida en la vejez avanzada de la que forma parte el estudio que se presenta en este trabajo, se seleccionaron encuestadoras a las que se formó para unificar las condiciones de recogida de información y para evitar la inducción de respuestas.

Utilizando los datos del censo, se seleccionaron aleatoriamente 100 hombres y 100 mujeres de dos subgrupos de edad establecidos a priori (75-84 y 85 y más). Ambos grupos fueron delimitados para asegurar la obtención de información de un grupo numeroso de personas más mayores $(85+)$, grupo que suele estar infrarrepresentado en los estudios por la dificultad de acceso a personas de esas edades. A todos los participantes se les envió una carta en la que se informaba del estudio y se solicitaba su participación en él. Se identificaron tres sustitutos de la misma edad y sexo para cada seleccionado previendo posibles bajas y la necesidad de reponer personas con similares características demográficas. Dada la elevada mortandad experimental que se produjo, en algunos casos hubo que completar la muestra con personas de la misma edad, más allá de los sustitutos. Entre las razones de la mortandad experimental figuran ausencia de interés propio en el estudio, fallecimiento de la persona seleccionada, traslado del domicilio sin haber dejado un domicilio alternativo y negativa de los familiares a que sus mayores colaborasen en el estudio. Hubo algunos casos en que la imposibilidad de participar afectó a los tres sustitutos seleccionados inicialmente, lo que hizo que se recurriera a personas de la misma edad y género, más allá de los sustitutos. En torno a un $25 \%$ de participantes tuvo que ser repuesto.

Días después del envío de la carta, las evaluadoras acudían al domicilio de las personas mayores, explicaban el objetivo del estudio y aseguraban el anonimato y la confidencialidad de sus respuestas. Tras obtener el consentimiento informado, se concertaba una cita en el propio domicilio.

\section{Análisis de datos}

Se clasificaron los problemas de salud referidos por las personas mayores y se calculó la distribución de frecuencias y de porcentajes de las categorías identificadas. Para verificar si en la vejez avanzada se emplean diferentes estrategias de afrontamiento para los problemas de salud, se calcularon las medias y desviaciones típicas de cada una de las estrategias y se ordenaron por rango. Además, se identificaron las asociaciones entre las estrategias mediante los coeficientes de correlación lineal de Pearson. Para comprobar si existen diferencias en función de la edad avanzada en el modo de afrontar los problemas de salud, se dividió a la muestra en dos grupos de edad, de 75 a 84 años y de 85 y más años, y se efectuaron análisis de varianza (ANOVAs). Finalmente, para conocer la contribución de las estrategias de afrontamiento sobre la autoeficacia en el manejo de los problemas de salud y sobre la satisfacción vital durante la vejez avanzada, se realizaron análisis de regresión múltiple simultánea. Se tomaron como criterios la autoeficacia en el manejo de los problemas y la satisfacción vital y, siguiendo la recomendación efectuada por Hosmer y Lemeshow (1989), se incluyeron como variables predictoras las que habían obtenido una correlación significativa con un nivel de confianza superior o igual a .95. Todos los análisis estadísticos se realizaron con el programa SPSS 19.0, aceptándose un nivel de significación del 95\%.

\section{Resultados}

En relación con los problemas de salud identificados, en la Tabla 2 se presenta la clasificación realizada sobre los estresores referidos por las personas muy mayores participantes en este estudio, así como la distribución de frecuencias y de porcentajes de las categorías identificadas.

Tabla 2. Problemas de salud referidos por los participantes y autoeficacia media (y desviación típica) para cada uno de ellos.

\begin{tabular}{llc}
\hline Problema & $\mathrm{N}(\%)$ & $\mathrm{M}(\mathrm{DT})$ \\
\hline Para caminar & $25(15.7)$ & $1.75(.73)$ \\
Fractura & $15(9.4)$ & $1.69(.63)$ \\
De espalda & $12(7.5)$ & $1.70(.77)$ \\
De visión & $12(7.5)$ & $1.30(.82)$ \\
Respiratorio & $11(6.9)$ & $1.91(.83)$ \\
Cardíaco & $11(6.9)$ & $2.13(.99)$ \\
Artrosis & $10(6.3)$ & $2(1)$ \\
Dolor inespecífico & $10(6.3)$ & $2.33(.70)$ \\
De salud sin especificar & $8(5)$ & $1.88(.83)$ \\
Mareos & $7(4.4)$ & $1.86(.69)$ \\
Circulatorio & $7(4.4)$ & $1.83(.40)$ \\
Digestivo & $6(3.8)$ & $1.60(.54)$ \\
Gripe & $6(3.8)$ & $2.20(.44)$ \\
Diabetes & $5(3.1)$ & $1.80(.44)$ \\
Urinario & $4(2.5)$ & $2.50(.70)$ \\
De sueño & $3(1.9)$ & $.67(.57)$ \\
De memoria & $3(1.9)$ & $1.67(.57)$ \\
De audición & $2(1.3)$ & $2(1.41)$ \\
De próstata & $2(1.3)$ & $2(0)$ \\
\hline
\end{tabular}

Tal como puede verse, las condiciones referidas son muy variadas; predominan claramente los problemas crónicos de salud, aunque también se indican problemas agudos. Asimismo, un 5\% responde genéricamente, sin especificar el problema de salud concreto.

En la Tabla 3 se ofrecen, ordenadas por rangos, las estrategias de afrontamiento referidas por las personas muy mayores para hacer frente a sus problemas de salud, junto 
con las medias y las desviaciones típicas de cada una de ellas.

Tabla 3. Descriptivos de las 18 estrategias de afrontamiento de los problemas de salud.

\begin{tabular}{lll}
\hline Rango & Estrategias de afrontamiento & $M(D T)$ \\
\hline 1 & Resolver el problema & $8.15(2.43)$ \\
\hline 3 & Desarrollo personal & $7.73(2.27)$ \\
& Conformismo & $7.38(2.53)$ \\
\hline 4 & Planificación & $7.27(2.43)$ \\
& Apoyo emocional & $7.07(2.65)$ \\
\hline 5 & Reevaluación positiva & $6.57(2.45)$ \\
& Expresión emocional & $6.53(2.31)$ \\
\hline 6 & Distanciamiento & $5.94(2.19)$ \\
& Refrenar el afrontamiento & $5.90(2.21)$ \\
& Desconexión cognitiva & $5.67(1.99)$ \\
& Apoyo social & $5.50(2.34)$ \\
\hline 7 & Reacción depresiva & $4.97(2.22)$ \\
\hline 8 & Supresión de actividades distractoras & $4.95(2.20)$ \\
\hline 10 & Evitar el afrontamiento & $4.66(1.99)$ \\
\hline & Control emocional & $4.35(2.32)$ \\
\hline & Desconexión comportamental & $3.80(2.13)$ \\
\hline & Respuesta paliativa & $3.13(2.08)$ \\
\hline
\end{tabular}

Como puede observarse, las personas muy mayores emplean diferentes estrategias para hacer frente a sus problemas de salud. La más utilizada es resolver el problema, estrategia de naturaleza activa y comportamental. Las estrategias desarrollo personal, planificación y reevaluación positiva, son estrategias también activas, aunque cognitivas, utilizadas con mucha frecuencia. En posiciones preferentes destacan dos estrategias pasivas, el conformismo, estrategia cognitiva centrada en el problema y el apoyo social emocional, estrategia comportamental centrada en la emoción.

Por el contrario, estrategias evitativas como la negación, respuesta paliativa y desconexión comportamental, son las menos utilizadas. Además, otras estrategias evitativas, tales como distanciamiento, desconexión cognitiva y evitar el afrontamiento son utilizadas ocasionalmente. Del mismo modo, estrategias pasivas como refrenar el afrontamiento, apoyo social, reacción depresiva y control emocional aparecen también en posiciones intermedias. Entre ellas y con la misma frecuencia de uso, se encuentra la estrategia de supresión de actividades distractoras, estrategia activa de carácter comportamental.

En la Tabla 4 se presenta la matriz de correlaciones lineales entre las 18 estrategias de afrontamiento. Los coeficientes de correlación obtenidos son de cuantía moderada, siendo el mayor de .71 .

Tal como puede apreciarse al observar dicha matriz, existe una gran cantidad de asociaciones significativas en el empleo de las distintas estrategias de afrontamiento. Cada estrategia se utiliza de manera combinada con otras, tanto de su misma naturaleza como de naturaleza diferente.
Sobre las diferencias de edad en el afrontamiento de los problemas de salud, únicamente se obtuvieron diferencias estadísticamente significativas en la utilización de las estrategias de desarrollo personal y reevaluación positiva. Las emplean más las personas de hasta 84 años (Medias de 7.09 frente a 6.11 y de 8.11 frente a 7.38 , respectivamente).

En cuanto a la efectividad en el manejo de los problemas de salud, las personas muy mayores se sienten bastante autoeficaces $(M=1.82 ; D T=.76)$. Siguiendo la recomendación de Hosmer y Lemeshow (1989) comentada anteriormente, en el análisis de regresión múltiple efectuado sobre esta variable criterio se incluyeron trece estrategias de afrontamiento: control emocional, distanciamiento, reacción depresiva, desconexión comportamental, resolver el problema, planificación, apoyo emocional, reevaluación positiva, refrenar el afrontamiento, expresión emocional, conformismo, desarrollo personal y negación. Dichas estrategias explican un $30 \%$ de la varianza en la percepción de autoeficacia $[F(13,141)=5.813, p<.001]$. Como muestra la Tabla 5, los resultados indican que planificación $(\beta=.309, p=.007)$, resolver el problema $(\beta=.267, p=.024)$, control emocional $(\beta=-.237, p=$ $.005)$ y reacción depresiva $(\beta=-.198, p=.014)$, son las estrategias que predicen significativamente la autopercepción de manejo efectivo de los problemas de salud en la vejez avanzada. Planificación y resolver el problema contribuyen positivamente, mientras que control emocional y reacción depresiva, lo hacen de modo negativo.

Tabla 5. Resultados del análisis de regresión de las estrategias de afrontamiento sobre la autoeficacia en el manejo de los problemas de salud y sobre la satisfacción vital.

\begin{tabular}{lccc}
\hline Predictores & $\beta$ & $t$ & $p$ \\
\hline Autoeficacia en el manejo & & & \\
Planificación & .30 & 2.75 & .007 \\
Resolver el problema & .26 & 2.27 & .024 \\
Reacción depresiva & -.19 & -2.47 & .014 \\
Control emocional & -.23 & -2.85 & .005 \\
Refrenar afrontamiento & .10 & 1.26 & .207 \\
Conformismo & .09 & 1.15 & .251 \\
Negación & .06 & .73 & .467 \\
Desconexión comportamental & .05 & .69 & .488 \\
Desarrollo personal & .05 & .60 & .249 \\
Expresión emocional & .04 & .49 & .619 \\
Distanciamiento & -.03 & -.45 & .649 \\
Apoyo emocional & -.04 & -.53 & .591 \\
Reevaluación positiva & -.05 & -.54 & .585 \\
$\quad$ Satisfacción vital & & & \\
Resolver el problema & .37 & 3.43 & .001 \\
Desconexión cognitiva & .12 & 1.43 & .15 \\
Refrenar afrontamiento & .03 & .40 & .68 \\
Apoyo emocional & .03 & 1.47 & .63 \\
Desconexión comportamental & -.08 & -1.00 & .31 \\
Planificación & -.07 & -.67 & .50 \\
Distanciamiento & -.06 & -.66 & .51 \\
\hline
\end{tabular}




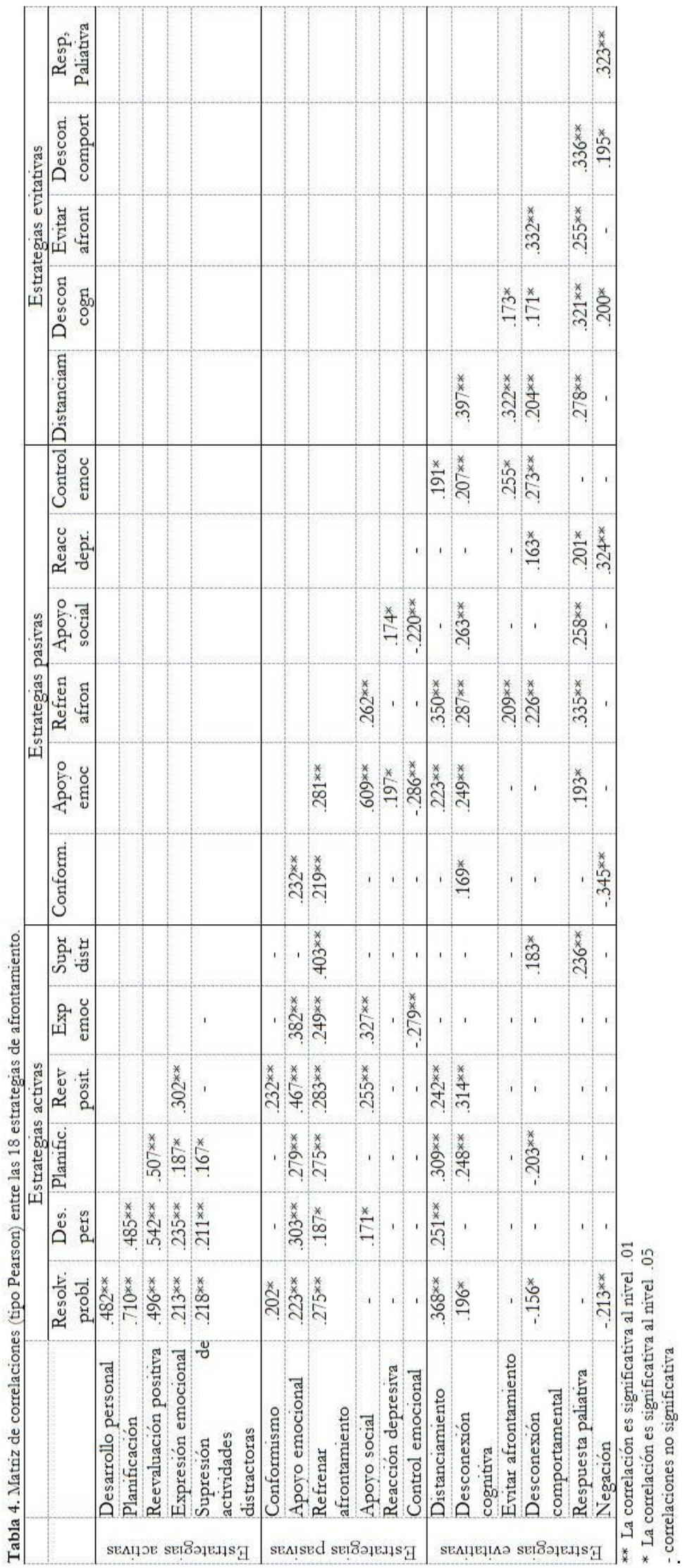


Por lo que se refiere a la satisfacción vital, las personas muy mayores indicaron encontrarse bastante satisfechas con su vida $(M=14.65 ; D T=3.07)$. En el análisis de regresión múltiple realizado utilizando la satisfacción vital como criterio se incluyeron las siete estrategias de afrontamiento siguientes: resolver el problema, desconexión cognitiva, refrenar el afrontamiento, apoyo emocional, desconexión comportamental, planificación y distanciamiento. El modelo explicó un $11 \%$ de la varianza en la satisfacción con la vida $[F(7,158)=3.787, p<.001]$. Tal como se detalla en la Tabla 5 , los resultados indican que resolver el problema $(\beta=.378, p<$ .001) es la única estrategia que contribuye significativamente sobre la satisfacción vital en la vejez avanzada.

\section{Discusión}

El objetivo de este estudio es analizar las estrategias de afrontamiento utilizadas por las personas muy mayores para hacer frente a sus problemas de salud más importantes, así como identificar la contribución de dichas estrategias sobre varios resultados de funcionamiento en la vejez avanzada. Las escasas diferencias de edad encontradas en el afrontamiento de los problemas de salud permiten considerar globalmente la muestra de edad avanzada.

La heterogeneidad en los problemas de salud referidos por los participantes, así como la presencia relativa desigual de los distintos tipos de problemas dentro de la muestra estudiada, impiden la identificación de perfiles de afrontamiento entre las personas muy mayores de este estudio. A pesar de ello, los resultados de este trabajo permiten afirmar que las personas de más de 75 años de nuestro entorno que viven en la comunidad utilizan un gran repertorio de estrategias de afrontamiento ante sus problemas de salud. La cantidad y variedad de estrategias de afrontamiento utilizadas puede ser reflejo de la flexibilidad que tienen las personas muy mayores, flexibilidad que se mantiene hasta las últimas etapas de la vida.

En este estudio, la mayor parte de los problemas de salud que las personas muy mayores tenían que afrontar eran discapacidades y enfermedades crónicas, aunque también han aparecido problemas agudos. Para hacerles frente, las personas de más de 75 años utilizan estrategias de afrontamiento que implican una valoración y una aproximación activa al problema. La estrategia más utilizada es de naturaleza activa y comportamental, resolver el problema, lo que significa que las personas muy mayores emplean muy a menudo acciones directas y racionales para solucionar sus situaciones de salud problemáticas. Otras estrategias activas, pero de naturaleza cognitiva, como desarrollo personal, planificación y reevaluación positiva, son utilizadas también con mucha frecuencia. Implican considerar los problemas de salud como un aprendizaje positivo, analizar racionalmente el problema de salud y crear un nuevo significado de la situación problema, intentando sacar todo lo positivo que tenga. Junto a ellas destaca otra estrategia activa de carácter comportamental, la expresión emocional, centrada en exteriorizar las emociones causadas por el problema de salud.

No obstante, junto a un afrontamiento predominantemente activo que moviliza comportamientos y cogniciones, tanto las personas muy mayores como las más mayores, recurren también a modos de afrontamiento pasivos, aceptando las consecuencias derivadas de su problema de salud y buscando apoyo y comprensión emocional para la situación en la que se encuentran inmersas. La utilización preferente del conformismo y del apoyo social emocional, junto a las asociaciones positivas entre ellas y las estrategias activas así lo ponen de manifiesto. Que las personas muy mayores confíen en estrategias pasivas puede resultar adaptativo dado que muchos de los problemas de salud referidos por las personas de este estudio eran irreversibles. Tal como sostenía Thomae (2002), que las personas mayores confíen en estrategias pasivas no significa que se resignen pasivamente a su problema de salud; significa convertir una situación no favorable e incontrolable en internamente aceptable.

Por otro lado, estrategias evitativas como la negación, la respuesta paliativa y la desconexión comportamental, son las menos utilizadas y algunas de ellas mantienen una relación inversa con las estrategias activas más empleadas. Este resultado es muy relevante puesto que pone de manifiesto que en la vejez avanzada apenas se utilizan estrategias "regresivas" caracterizadas por no aceptar el problema, por ayudarse de elementos que busquen evitar el problema de salud, por ejemplo, buscar sentirse mejor viendo la televisión o comiendo, y por la evitación de cualquier tipo de respuesta o solución al problema. Además, resultan incompatibles con estrategias activas muy utilizadas. Este patrón de afrontamiento es consistente con los obtenidos por Diehl, Coyle y Labouvie-Vief (1996) y por Vaillant (2000), quienes defienden la existencia de un afrontamiento "maduro" de los problemas en la vejez, en la medida en que se utilizan más estrategias como la reinterpretación positiva o la aceptación y menos mecanismos como la negación o las respuestas paliativas.

Sin embargo, algunas estrategias evitativas, como el distanciamiento y la desconexión cognitiva, son utilizadas ocasionalmente vinculadas a estrategias activas de uso preferente. Esto significa que las personas muy mayores en algunos momentos se alejan emocionalmente del problema de salud, piensan en otras cosas que les impidan centrarse en él y no hacen nada por miedo a empeorarlo o por considerarlo irresoluble. Del mismo modo, estrategias pasivas como refrenar el afrontamiento, apoyo social, reacción depresiva y control emocional aparecen también en posiciones intermedias. Aplazar todo tipo de afrontamiento hasta obtener más información, y buscar apoyo y consejo sobre cómo resolver el problema de salud se utilizan conjuntamente con estrategias activas muy frecuentemente utilizadas. También en posiciones intermedias se encuentra la estrategia de supresión de actividades distractoras, estrategia activa de carácter comportamental, que se refiere a paralizar todo tipo de actividades para centrarse activamente en la búsqueda de información con el fin de valorar el pro- 
blema de salud. Estos resultados apoyan la presencia de estrategias de control primario y secundario para hacer frente a los problemas de salud en edades avanzadas, tal como sostienen Heckhausen, Wrosch y Schulz (2010).

Todo lo anterior indica que las personas muy mayores presentan un patrón de afrontamiento frente a sus problemas de salud en el que está presente fundamentalmente lo activo, pero también lo pasivo y, en mucha menor medida, lo evitativo. Esto puede significar que, en la mayoría de los casos, la persona muy mayor intenta modificar las circunstancias de su problema de salud que le apartan o dificultan el logro de sus metas y preferencias personales, movilizando esfuerzos para solucionarlo, pero también se conforma porque la solución definitiva a su problema no existe o la percibe como fuera de su alcance, con lo que busca apoyo y comprensión en los demás y trata de suprimir cognitivamente los efectos emocionales para no sentirse desbordada. En línea con estos hallazgos, investigadores como Brandtstädter y Rothermund (2002) y Boerner (2004) hablan del interjuego entre estrategias asimilativas y acomodativas en la vejez.

Respecto a las escasas diferencias de edad encontradas en el uso de las estrategias de afrontamiento frente a los problemas de salud, es probable que en la vejez los repertorios de afrontamiento estén desarrollados por completo, razón por la cual no aparezcan diferencias de edad. Tanto las personas muy mayores como las más mayores aplican, ante sus problemas de salud, los modos de afrontamiento que se han mostrado más eficaces en el pasado ante problemas similares. A través de su experiencia han aprendido qué tipo de estrategias son inefectivas y cuáles pueden ayudarles a lograr su meta en las situaciones problemáticas. Estos resultados son bastante consistentes con los obtenidos por Aldwin et al. (1996) al comparar el afrontamiento de personas maduras, mayores y muy mayores y con los encontrados por Martin et al. (2001) tras comparar sexagenarios, octogenarios y centenarios.

En cuanto a las consecuencias inmediatas del afrontamiento, los resultados de este trabajo indican que, aunque en la vejez avanzada las personas utilizan estrategias de diferente naturaleza, no todas ellas tienen el mismo valor adaptativo. Únicamente algunas estrategias activas contribuyen positivamente sobre la percepción de habilidad personal para hacer frente a las situaciones de salud problemáticas, mientras que las estrategias pasivas y evitativas que determinan la autoeficacia de afrontamiento contribuyen negativamente sobre ella. El sentido de competencia personal ante los problemas de salud en la vejez avanzada sólo se asocia positivamente con una aproximación analítica y racional al problema y con acciones directas y racionales para resolverlo, mientras que regular y ocultar los sentimientos generados por el problema y sentirse desbordados por él hacen que disminuya dicho sentido de competencia. Por lo tanto, solamente las estrategias activas generan un sentido positivo de autoeficacia en la superación de los problemas de salud en la vejez avanzada, con las implicaciones que eso puede tener sobre el sentido de control interno, así como sobre las emociones, el esfuerzo y la persistencia para el logro de los objetivos personales (Ar- tistico, Cervone y Pezzuti, 2003; Löffler et al., 2012; Moskowitz et al., 2012; Schwarzer y Renner, 2000).

Un último aspecto relevante de este trabajo lo constituye la relación del afrontamiento con la satisfacción vital en tanto que resultado de funcionamiento. Del mismo modo que en otros trabajos previos (p. ej., Smith, Fleeson, Geiselmann, Settersten y Kunzmann, 1999; Villar, Triadó, Solé y Osuna, 2003), las personas muy mayores se encuentran bastante satisfechas con su vida. Esto sugiere que en la vejez avanzada la satisfacción vital no es menor que en otras etapas, es decir, las personas muy mayores todavía son capaces de adaptarse a las circunstancias vitales cambiantes para mantener la satisfacción con uno mismo y con la vida. Ante los problemas de salud, la realización de acciones directas y racionales para resolverlos ha manifestado su funcionalidad en el mantenimiento de la satisfacción vital de las personas de más de 75 años. Por lo tanto, dicha estrategia resulta protectora frente al estrés que acompaña a los problemas de salud. Este resultado es consistente con estudios previos que muestran la asociación entre utilizar acciones instrumentales para afrontar las dificultades con el bienestar y otros resultados positivos (Jonker, Comijs, Knipscheer y Deeg, 2009; Moos et al., 2006). No obstante, el reducido porcentaje de varianza en satisfacción vital explicado por el conjunto de las estrategias de afrontamiento resulta indicativo de un impacto limitado de éstas sobre aquélla y apunta hacia la naturaleza multidimensional de un índice global que se nutre de la contribución de múltiples determinantes (Wu et al., 2013).

Tomados conjuntamente, los hallazgos de este trabajo no apoyan la idea de que un afrontamiento centrado en el problema sea desadaptativo para las personas muy mayores, dados los recursos limitados y la naturaleza intratable de muchos de sus problemas (Isaacowitz y Seligman, 2001, 2002; Wrosch, Heckhausen y Lachman, 2000). Por el contrario, frente a los estudios que se han centrado en la depresión en vez de en el bienestar y en los resultados positivos de los sucesos (p. ej., autoeficacia), nuestros hallazgos apoyan la idea de un proceso de congruencia afrontamiento-resultado en el que el afrontamiento de aproximación al problema se asocia estrechamente con resultados positivos de funcionamiento, mientras que el afrontamiento pasivo y evitativo se asocia con resultados negativos (menor autoeficacia de afrontamiento).

La adaptación al envejecimiento es un proceso dinámico en el que la persona se enfrenta a problemas y a cambios asociados a su envejecimiento, no como una víctima pasiva, sino como un actor que recurre a diferentes estrategias que le lleven a cambios que pueden ser positivos y negativos. Se constata que el estudio del afrontamiento de los problemas de salud es un tema relevante que merece ser trabajado por el papel que desempeña sobre indicadores importantes de adaptación en la vejez avanzada.

El alcance y generalizabilidad de los resultados y conclusiones de este trabajo quedan limitados por algunas cuestiones. En primer lugar, los problemas de salud identificados han resultado muy heterogéneos lo que ha podido afectar al 
patrón de afrontamiento obtenido; conseguir muestras de personas muy mayores que compartan los mismos tipos de problemas de salud puede ayudar a clarificar mejor las características contextuales del afrontamiento. Por otra parte, no se ha controlado la severidad de los problemas referidos ni la evaluación subjetiva de los mismos, variables que pueden ser importantes en relación con el tema de estudio. Tampoco se ha examinado el papel que puede tener sobre la salud y las estrategias de afrontamiento el hecho de que los sujetos vivan solos o en pareja. Considerar estos factores en el diseño de futuros trabajos permitiría analizar en mayor profundidad las características del afrontamiento y la naturaleza del mismo en personas muy mayores, hecho que contribuirá a aumentar el escaso conocimiento actual sobre el manejo de los estresores más prevalentes en la vejez avanzada.

Los datos obtenidos en este trabajo suponen una primera aproximación al afrontamiento de los problemas de salud por parte de las personas muy mayores y a su valor más o menos adaptativo en relación con determinados resultados positivos de funcionamiento. Este hecho potencia la situa-

\section{Referencias}

Aldwin, C.M. (2007). Stress, coping, and development: An integrative approach. New York, NY: Guilford.

Aldwin, C.M., \& Park, C.L. (2004). Coping and physical health outcomes: an overview. Psychology and Health, 19, 277-281.

Aldwin, C.M., Sutton, K.J., Chiara, G., \& Spiro, A. (1996). Age differences in stress, coping and appraisal: findings from the normative aging study. Journal of Gerontology, 51, 179-188.

Artistico, D., Cervone, D., \& Pezzuti, L. (2003). Perceived self-efficacy and everyday problem solving among young and older adults. Psychology and Aging, 18, 68-79.

Baltes, P.B., \& Mayer, K.U. (1999). The Berlin Aging Study: Aging from 70 to 100. New York, NY: Cambridge University Press.

Boerner, K. (2004). Adaptation to disability among middle-aged and older adults: The role assimilative and accommodative coping. Journal of Gerontology: Psychological Sciences, 54, S230-237.

Brandtstädter, J., \& Rothermund, K. (2002). The life-course dynamics of goal pursuit and goal adjustment: A two-process framework. Developmental Review, 22, 117-150.

Carver, C.S. (2004). Negative affects deriving from the behavioural approach system. Emotion, 4, 3-22.

Chida, Y., \& Hamer, M. (2008). Chronic psychosocial factors and acute physiological responses to laboratory-induced stress in healthy populations: a quantitative review of 30 years of investigations. Psychological Bulletin, 134, 829-885.

Diehl, M., Coyle, N., \& Labouvie-Vief, C. (1996). Age and sex differences in strategies of coping and defense across the life span. Psychology and $A g$ ing, 11, 127-139.

Diener, E., Emmons, R.A., Larsen, R.J., \& Griffin, S. (1985). The satisfaction with life scale. Journal of Personality Assessment, 49, 71-75.

Diener, E., Ng., W., Harter, J., \& Arora, R. (2010). Wealth and happiness across the world: Material prosperity predicts life evaluation, while psychosocial prosperity predicts positive feeling. Journal of Personality and Social Psychology, 97, 143-156.

Dunkel-Schetter, C., Feinstein, L.G., Taylor, S.E., \& Falke, R.L. (2000). Patterns of coping with cancer. En R.M. Suin \& G.R. VandenBos (Eds.). Cancer patients and their families: Readings on disease course, coping, and psychological interventions (pp. 35-51). Washington D.C.: American Psychological Association.

Fernández-Abascal, E.G. (1997). Estilos y estrategias de afrontamiento. En E.G. Fernández-Abascal, (Ed.), Cuadernos de prácticas de motivación y emoción (pp. 189-206). Madrid, España: Pirámide. ción del afrontamiento como factor de resiliencia psicológica en la vejez avanzada, en línea con la argumentación de otros autores (Baltes y Mayer, 1999). En todo caso, este estudio contribuye a justificar la necesidad y pertinencia de poner en marcha programas de intervención para enseñar a los ancianos las diferencias entre distintas habilidades de afrontamiento, resaltando el valor positivo de las estrategias activas de afrontamiento y la posibilidad de encontrar beneficios al manejar diferentes estresores de salud. Los programas de intervención sobre el afrontamiento de problemas de salud deberían ser evaluados no sólo sobre la base de si reducen o no el estrés de las personas mayores, sino también sobre cuáles son sus beneficios y perjuicios potenciales en relación con la efectividad en el manejo de los problemas y con la satisfacción a que puedan dar lugar.

Agradecimientos.- Las autoras agradecen los comentarios y sugerencias de los revisores, que han permitido mejorar la calidad de este trabajo.

Franks, H.M., \& Roesch, S.C. (2006). Appraisals and coping in people living with cancer: A meta-analysis. Psycho-Oncology, 15, 1027-1037.

Hamarat, E., Thompson, D., Zabrucky, K.M., Steele, D., \& Matheny, K.B. (2001). Perceived stress and coping resource availability as predictors of life satisfaction in young, middle-aged, and older adults. Experimental Aging Research, 27, 181-196.

Harnish, J.D., Aseltine, R.H., \& Gore, S. (2000). Resolution of stressful experiences as an indicator of coping effectiveness in young adults: An event history analysis. Journal of Health and Social Behavior, 41, 121-136.

Heckhausen, J., Wrosch, C., \& Schulz, R. (2010). A motivational theory of lifespan development. Psychological Review, 117, 32-60.

Holland, K.D., \& Holahan, C.K. (2003). The relation of social support and coping to positive adaptation to breast cancer. Psychology and Health, 18, 15-29.

Hosmer, D.W., \& Lemeshow, S. (1989). Applied logistic regression. New York, NY: John Wiley \& Sons.

Isaacowitz, D.M., \& Seligman, M.E.P. (2001). Is pessimism a risk factor for depressive mood among community-dwelling older adults? Behaviour Research and Therapy, 39, 255-272.

Isaacowitz, D.M., \& Seligman, M.E.P. (2002). Cognitive style predictors of affect change in older adults. International Journal of Aging and Human Development, 54, 233-253.

Jonker, A.A., Comijs, H.C., Knipscheer, K.C., \& Deeg, D.J. (2009). The role of coping resources on change in well-being during persistent health decline. Journal of Aging and Health, 21, 1063-1082.

Kraaij, V., Garnefski, N., \& Maes, S. (2002). The joint effects of stress, coping, and coping resources on depressive symptoms in the elderly. Anxiety, Stress, and Coping, 15, 163-177.

Kraaij, V., Pruymboom, E., \& Garnefski, N. (2002). Cognitive coping and depressive symptons in the elderly: a longitudinal study. Aging and Mental Health, 6, 275-281.

Löffler, C., Kaduszkiewicz, H., Stolzenbach, C., Streich, W., Fuchs, A., van den Bussche, H., Stolper, F., \& Altiner, A. (2012). Coping with multimorbility in old age - a qualitative study. BMC Family Practice, 13, 45.

Martin, P., Rott, C., Poon, L.W., Courtenay, B., \& Lehr, U. (2001). A molecular view of coping behavior in older adults. Journal of Aging and Health, 13, 72-91.

McKenna, M.C., Zevon, M.A., Corn, B., \& Rounds, J. (1999). Psychosocial factors and the development of breast cancer: A meta-analysis. Health Psychology, 18, 520-531. 
Meléndez, J.C., Mayordomo, T., Sancho, P. y Tomás, J.M. (2012). Coping strategies: Gender differences and development throughout Life Span. The Spanish Journal of Psychology, 15, 1089-1098.

Montero, I. y León, O.G. (2005). Sistema de clasificación del método en los informes de investigación en Psicología. International Journal of Clinical and Health Psychology, 5, 115-127.

Montorio, I., Nuevo, R., Márquez, M., Izal, M. y Losada, A. (2003). Characterization of worry according to severity of anxiety in elderly living in the community. Aging and Mental Health, 7, 334-341.

Moos, R.H., Brennan, P.L., Schutte, K.K., \& Moos, B.S. (2006). Older adults'coping with negative life events: common processes of managing health, interpersonal, and financial/work stressors. International Journal of Aging and Human Development, 62, 39-59.

Moskowitz, J.T., Hult, J.R., Duncan, L.G., Cohn, M.A., Maurer, S., Bussolari, C., \& Acree, M. (2012). A positive affect intervention for people experiencing health-related stress: Development and non-randomized pilot test. Journal of Health Psychology, 17, 676-692.

Ong, A.D., Bergeman, C.S., \& Boker, S.M. (2010). Resilience comes of age : Defining features in later adulthood. Journal of Personality, 77, 1777-1804.

Penley, J.A., Tomaka, J., \& Wiebe, J.S. (2002). The association of coping to physical and psychological health outcomes: A meta-analytic review. Journal of Behavioral Medicine, 6, 551-603.

Prokopcakova, A. (2004). Choice of coping strategies in the interaction: anxiety and type of a demanding life situation (a research probe). Studia Psychologica, 46, 235-238.

Revenson, T.A., Abraído-Lanza, A.F., Majerovitz, S.D., \& Jordan, C. (2005). Couples coping with chronic illness: What's gender got to do with it? En T.A. Revenson, K. Kayser, \& G. Bodenmann (Eds.), Couples coping with stress: Emerging perspectives on dyadic coping (pp. 137-156). Washington DC: American Psychological Association.

Rothermund, K., \& Brandtstädter, J. (2003). Depression in later life: crosssequential patterns and possible determinants. Psychology and Aging, 18, 80-90.

Smith, J., Fleeson, W., Geiselmann, B., Settersten Jr., R.A., \& Kunzmann, U. (1999). Sources of well-being in very old age. En P.B. Baltes, \& K.U. Mayer (Eds.), The Berlin Aging Study: Aging from 70 to 100 (pp. 450-471). New York, NY: Cambridge University Press.

Sonnenberg, C.M., Deeg, D.J., van Tilburg, T.G., Vink, D., Stek, M.L., \& Beekman, A.T. (2013). Gender differences in the relation between depression and social support in later life. International Psychogeriatrics, 25, 61-70.
Staudinger, U.M.; Freund, A.M.; Linden, M., \& Maas, I. (1999). Self, personality, and life regulation: Facets of psychological resilience in old age. En P.B. Baltes y K.U. Mayer (Eds.), The Berlin aging study: Aging from 70 to 100 (pp. 302-328). New York, NY: Cambridge University Press.

Schwarzer, R., \& Renner, B. (2000). Social-cognitive predictors of health behaviour: Action self-efficacy and coping self-efficacy. Health Psychology, 19, 487-49.

Taylor, S.E., \& Stanton, A.L. (2007). Coping resources, coping processes, and mental health. Annual Review of Clinical Psychology, 3, 377-401.

Thomae, H. (2002). Haciéndole frente al estrés en la vejez. Revista Latinoamericana de Psicología del Ciclo Vital, Envejecimiento y Vejez, 34, 41-54.

Thompson, R.J., Mata, J., Jaeggi, S.M., Buschkuekl, M., Jonides, J., \& Gotlib, I.H. (2010). Maladaptive coping, adaptive coping, and depressive symptoms: Variations across age and depressive state. Behavioral Research Theo$r y, 48,459-466$.

Tobin, D.J., Holroyd, K., Reynolds, R.V. \& Wigal, J.K. (1989). The hierarchical factor structure of the Coping Strategies Inventory. Cognitive Therapy and Research, 13, 343-361.

UNFPA (2012). Ageing in the twenty-first century: A celebration and a challenge. New York, NY: UNFPA.

Urcuyo, K.R., Boyers, A.E., Carver, C.S., \& Antoni, M.H. (2005). Finding benefit in breast cancer: Relations with personality, coping, and concurrent well-being. Psychology and Health, 20, 175-192.

Vaillant, G.E. (2000). Adaptive mental mechanisms: Their role in a positive psychology. American Psychologist, 55, 89-98.

Villar, F., Triadó, C., Solé, C. y Osuna, M.J. (2003). Bienestar, adaptación y envejecimiento: Cuando la estabilidad significa cambio. Revista Multidisciplinar de Gerontología, 13, 152-162.

Wu, G., Feder, A., Cohen, H., Kim, J.J., Calderon, S., Charney, D.S., \& Mathé, A.A. (2013). Understanding resilience. Frontiers in Behavioral Neurosciences, 7,10 .

Wrosch, C., Heckhausen, J., \& Lachman, M.E. (2000). Primary and secondary control strategies for managing health and financial stress across adulthood. Psychology and Aging, 15, 387-399.

Yancura, L., \& Aldwin, C. (2008). Coping and health in older adults. Current Psychiatry Reports, 10, 5-10.

Zaza, C., Sellick, S.M., \& Hillier, L.M. (2005). Coping with cancer: What do patients do. Journal of Psychosocial Oncology, 23, 55-73.

(Articulo recibido: 08-04-2013; revisado: 19-06-2014; aceptado: 06-07-2014) 